\title{
Effects of Morphology and Topology on the Effective Stiffness of Chiral Cellular Materials in the Transverse Plane
}

\author{
Alp Karakoç and Ertuğrul Taciroğlu \\ Civil and Environmental Engineering Department, University of California, Los Angeles, CA 90095, USA \\ Correspondence should be addressed to Alp Karakoç; akarakoc@ucla.edu
}

Received 10 April 2016; Accepted 2 June 2016

Academic Editor: Ying Li

Copyright (C) 2016 A. Karakoç and E. Taciroğlu. This is an open access article distributed under the Creative Commons Attribution License, which permits unrestricted use, distribution, and reproduction in any medium, provided the original work is properly cited.

\begin{abstract}
The present study investigates the influence of topology and morphology on the effective stiffness of chiral cellular materials in the transverse plane by means of a homogenization method. For this purpose, finite element models of representative volume elements for regular hexagonal and hexagonal-chiral configurations are used and simulations are conducted to quantify how cell topology-that is, chirality inside the cell-and cell wall slenderness affect the effective stiffness. Closed form solutions for regular hexagonal square and triangular RVEs provided in the literature are then taken as a basis for model validation. The results indicate that there are drastic differences between regular hexagonal and hexagonal-chiral configurations, which can be explained in terms of deformation mechanism transformations between bending and stretching. The investigations also reveal the positive impact of cell wall slenderness on stiffness due to volumetric increase in the cell wall material resisting the deformation.
\end{abstract}

\section{Introduction}

In recent years, with growing interest in energy efficient, robust, and lightweight material systems, cellular materials have found their way into numerous engineering applications. They are nowadays used as high-stiffness sandwich panels, energy absorbers, thermal insulators, and vibration dampers. Due to their low densities and high potential as being tailored materials in terms of effective stiffness and strength properties, there are ongoing research and development activities on these materials [1-3].

Dependence of these effective properties on cell wall geometrical, topological, and mechanical properties has been investigated in the framework of computational homogenization methods $[4,5]$. The framework is used to solve the associated boundary value problem with proper boundary conditions on representative volume elements (RVEs) that capture the prominent features of the material [6]. In the case of cellular materials, the term RVE refers to an individual cell or a group of cells, as depicted in Figure 1, that are assumed to represent the effective material properties at the mesoscale.

As a contribution to the current state of the art on cellular material characterization in the framework of computational homogenization, the current study aims at (i) understanding the effect of cell topology on the effective stiffness properties of cellular materials in the transverse plane by using regular hexagonal and hexagonal-chiral RVEs shown in Figure 1, (ii) understanding the effect of cell wall slenderness-that is, cell wall thickness-to-height ratio-on these effective properties, and (iii) comparing the extracted stiffness properties with analytical results given in the literature.

\section{Materials and Methods}

Computational homogenization has been a useful tool for determining the effective properties of materials that can be described through their microscopic properties. The basic idea behind this methodology is to evaluate the effective properties by solving the boundary value problem with appropriate boundary conditions defined on a representative volume element (RVE), which contains all necessary information $[7,8]$. Some of the key advantages include being able to introduce all of the necessary geometrical and constitutive information at appropriate scales of the material behavior and being able to use requisitely detailed yet computationally 

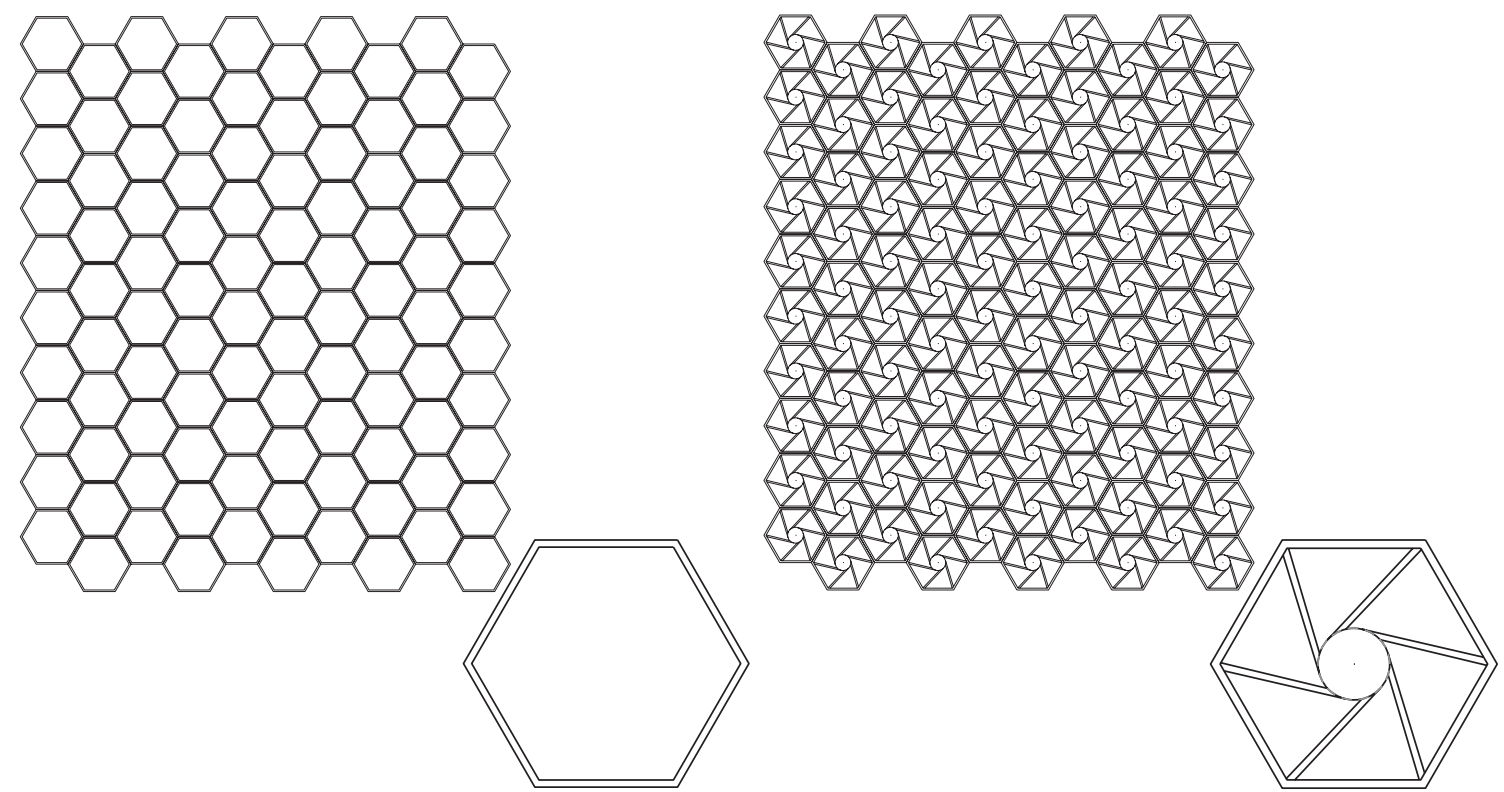

FIGURE 1: Hexagonal and hexagonal-chiral structures and corresponding representative volume elements.

effective idealization - such as beam and plate models - at the scale of the material cells $[9,10]$.

In the literature, numerous analytical and computational approaches have been offered to the homogenization problems involving cellular materials (see, e.g., [11-13]). Due to advances in theory and hardware, computational homogenization methods have become more frequently used for material characterization. In the present study, a computational homogenization method is thus used to investigate the effective stiffness properties of cellular materials, which is explained in detail in the following section.

2.1. A First-Order Strain-Driven Computational Homogenization Method. In the homogenization approach adopted here, the macroscopic strain $e_{i j}^{\mathrm{M}}$ for $i, j \in\{X, Y\}$ is assumed to be uniform over the material and imposed at the RVE boundary $\partial \omega$ as illustrated in Figure 2. Therefore, a first-order microscopic displacement field decomposition for the RVE can be given as

$$
\vec{u}^{\mathrm{m}}=\vec{r} \cdot \mathrm{e}^{\mathrm{M}}+\vec{u}
$$

for which the first term on the right-hand side represents the macroscopic displacement contribution and the second one represents the displacement fluctuation field $\vec{u}$ due to heterogeneities within the RVE [4]. Here, $\vec{r}$ represents the position vector with respect to any described origin.

Since the overall body is assumed to be composed of periodic arrays of repeating RVEs, continuity conditions for the displacements and tractions must be satisfied at the boundaries of each neighbouring cell. The first condition can be satisfied by taking the relative positions of the node sets, for example, nodes at the opposite edges on $\partial \omega$, for which

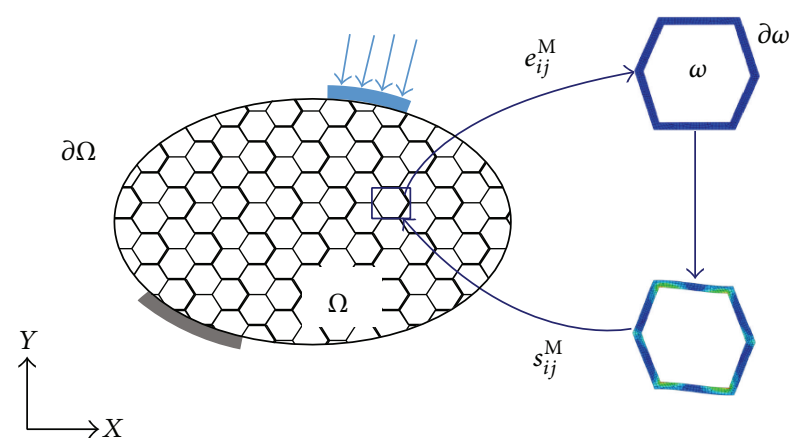

FIGURE 2: Strain-driven homogenization scheme with imposed macroscopic strain $e_{i j}^{\mathrm{M}}$ and computed stress $s_{i j}^{\mathrm{M}}$ for $i, j \in\{X, Y\}$. Here, $\Omega$ and $\partial \Omega$ represent the volume and boundary of aggregate, and $\omega$ and $\partial \omega$ represent the volume and boundary of RVE.

displacement fluctuations have the same value due to the periodicity condition [14]. Hence, (1) can be expanded as

$$
\vec{u}_{1}^{\mathrm{m}}-\vec{u}_{2}^{\mathrm{m}}=\left(\vec{r}_{1}-\vec{r}_{2}\right) \cdot \mathbf{e}^{\mathrm{M}},
$$

which forms the basis of the displacement boundary condition for periodic arrays. The traction boundary condition is satisfied with antiperiodicity of traction field in case of existence of traction on the boundaries [6]. However, the current study focuses on the displacement boundary conditions; therefore, only (2) is studied for the periodic boundary conditions.

In order to establish a consistent relationship between different scales, the Hill-Mandel principle can be used such that [15]

$$
\mathbf{s}^{\mathrm{M}}: \mathbf{e}^{\mathrm{M}}=\frac{1}{\omega} \int_{\omega} \mathbf{s}^{\mathrm{m}}: \mathbf{e}^{\mathrm{m}} \mathrm{d} \omega
$$


for which the superscript " $\mathrm{m}$ " represents the microscopic or RVE scale. The symbol ":” denotes an inner product, and for second-order tensors it is defined as

$$
\mathbf{a}: \mathbf{b}=a_{i j} b_{i j}
$$

By using Gauss's theorem, (3) can be rewritten at the RVE boundary $\partial \omega$ as

$$
\mathbf{s}^{\mathrm{M}}: \mathbf{e}^{\mathrm{M}}=\frac{1}{\omega} \int_{\partial \omega} \vec{t}^{\mathrm{m}} \cdot \vec{u}^{\mathrm{m}} \mathrm{d} \partial \omega
$$

where $\vec{t}^{\mathrm{m}}$ is the microscopic traction vector at $\partial \omega$. By plugging (1) into (5),

$$
\mathbf{s}^{\mathrm{M}}: \mathbf{e}^{\mathrm{M}}=\frac{1}{\omega} \int_{\partial \omega} \vec{t}^{\mathrm{m}} \cdot\left(\vec{r} \cdot \mathbf{e}^{\mathrm{M}}\right) \mathrm{d} \partial \omega+\frac{1}{\omega} \int_{\partial \omega} \vec{t}^{\mathrm{m}} \cdot \vec{u} \mathrm{~d} \partial \omega,
$$

which yields to

$$
\begin{aligned}
\mathbf{s}^{\mathrm{M}}: \mathbf{e}^{\mathrm{M}}= & \frac{1}{\omega} \int_{\partial \omega}\left(\vec{t}^{\mathrm{m}} \otimes \vec{r}\right) \mathrm{d} \partial \omega: \mathbf{e}^{\mathrm{M}} \\
& +\frac{1}{\omega} \int_{\partial \omega} \vec{t}^{\mathrm{m}} \cdot \vec{u} \mathrm{~d} \partial \omega .
\end{aligned}
$$

Here, the symbol $\otimes$ denotes a dyadic product. The second integrand at the right-hand side vanishes in case of periodic boundary conditions, as elaborated in (2). Hence, the macroscopic stress $s_{i j}^{\mathrm{M}}$ can be expressed as the volume average of the microscopic stress $s_{i j}^{\mathrm{m}}$ such that

$$
\mathbf{s}^{\mathrm{M}}=\frac{1}{\omega} \int_{\partial \omega}\left(\vec{t}^{\mathrm{m}} \otimes \vec{r}\right) \mathrm{d} \partial \omega=\frac{1}{\omega} \int_{\omega} \mathbf{s}^{\mathrm{m}} \mathrm{d} \omega,
$$

where $\omega$ is the total volume of the RVE.

Thereafter, the effective stiffness can be determined by means of the relationship between the macroscopic strain $e_{i j}^{\mathrm{M}}$ and stress $s_{i j}^{\mathrm{M}}$ under the linear elasticity assumption. This relationship can be constructed in terms of macroscopic compliance so that [16]

$$
\mathbf{e}^{\mathrm{M}}=\mathrm{C}^{\mathrm{M}}: \mathbf{s}^{\mathrm{M}} \text {. }
$$

Then, the Voigt notation for $\mathrm{C}^{\mathrm{M}}$ can be constructed as

$$
\left[\mathbf{C}^{\mathrm{M}}\right]=\left[\begin{array}{ccc}
e_{X X}^{\mathrm{M}, 1} & e_{X X}^{\mathrm{M}, 2} & e_{X X}^{\mathrm{M}, 3} \\
e_{Y Y}^{\mathrm{M}, 1} & e_{Y Y}^{\mathrm{M}, 2} & e_{Y Y}^{\mathrm{M}, 3} \\
2 e_{X Y}^{\mathrm{M}, 1} & 2 e_{X Y}^{\mathrm{M}, 2} & 2 e_{X Y}^{\mathrm{M}, 3}
\end{array}\right]\left[\begin{array}{ccc}
s_{X X}^{\mathrm{M}, 1} & s_{X X}^{\mathrm{M}, 2} & s_{X X}^{\mathrm{M}, 3} \\
s_{Y Y}^{\mathrm{M}, 1} & s_{Y Y}^{\mathrm{M}, 2} & s_{Y Y}^{\mathrm{M}, 3} \\
s_{X Y}^{\mathrm{M}, 1} & s_{X Y}^{\mathrm{M}, 2} & s_{X Y}^{\mathrm{M}, 3}
\end{array}\right]^{-1},
$$

for which $\left[\mathbf{C}^{\mathrm{M}}\right]$ is taken to be symmetric matrix. Superscripts 1,2 , and 3 represent three different loading conditions fulfilling the minimum requirement to obtain the compliance matrix. Under the assumption of orthotropic material properties,

$$
\left[\mathbf{C}^{\mathrm{M}}\right]=\left[\begin{array}{ccc}
\frac{1}{E_{X}} & \frac{-v_{Y X}}{E_{Y}} & 0 \\
\frac{-v_{X Y}}{E_{X}} & \frac{1}{E_{Y}} & 0 \\
0 & 0 & \frac{1}{G_{X Y}}
\end{array}\right]
$$

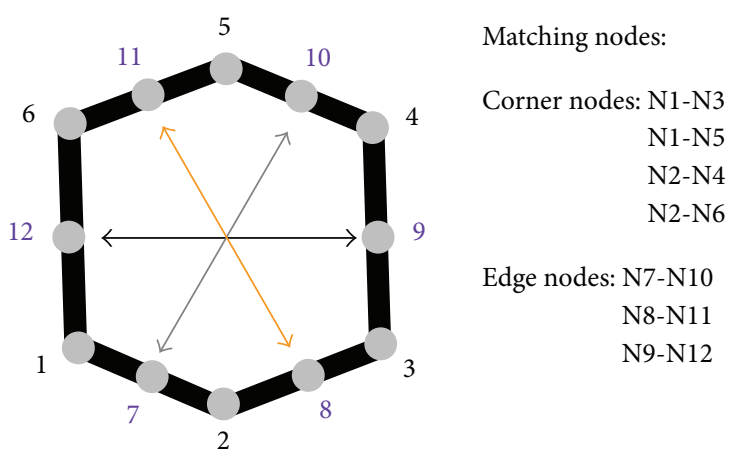

(a)

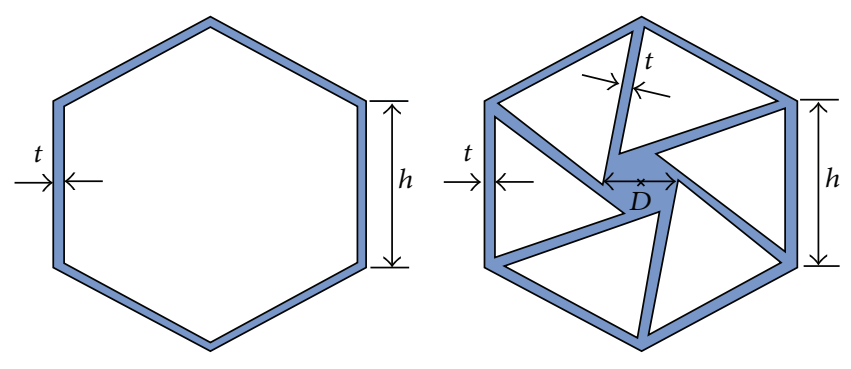

(b)

FIGURE 3: Schematics of the investigated geometrical properties and matching nodes for periodic boundary conditions: (a) nodal mapping for periodic boundary conditions and (b) cell geometric parameters, cell wall thickness $t$, height $h$, and hub diameter $D$.

for which $E_{X}$ and $E_{Y}$ are the effective Young's moduli, $G_{X Y}$ are the shear modulus, and $v_{X Y}$ and $v_{Y X}$ are Poisson's ratios with reciprocal relation $v_{X Y} / E_{X}=v_{Y X} / E_{Y}$ [17].

2.2. Implementation of Computational Homogenization. In this study, regular hexagonal and hexagonal-chiral RVEs are investigated. Therefore, the aforementioned equation sets are applied on matching node sets that form the periodicity conditions. The mapping is illustrated on a regular hexagonal RVE geometry as seen in Figure 3(a), whereas the geometrical parameters of regular hexagonal and hexagonal-chiral RVEs are depicted in Figure 3(b).

\section{Results and Discussion}

3.1. Design of Experiments. In the present study, cell walls are taken to be transversely isotropic under the assumption of uniform distribution of cell wall material in the plane of interest. Mechanical parameters of the cell walls are selected as $E_{s}=1000 \mathrm{~Pa}$ and $v_{s}=0.4$, which refer to typical cell wall Young's modulus and Poisson's ratio value, respectively. In relation to (9) and (10), three different in-plane loading conditions-that is, tensile load along the $X$ - and $Y$-directions and the $X Y$-shear load as listed in Table 1 and illustrated in Figure 4-are used to compute the effective stiffness properties and understand the effect of cell wall slenderness $\underline{t} / h$, for which $\underline{t}=2 t, t$ is cell wall thickness, and $h$ refers to cell wall height (as depicted in Figure 3), on the computed properties. The reason for taking the cell wall slenderness as $\underline{t}=2 t$ is 


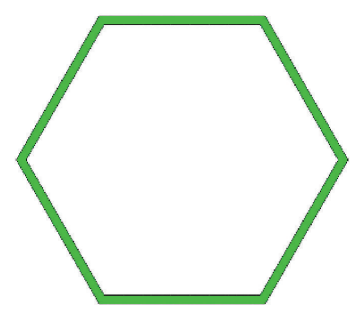

(a)

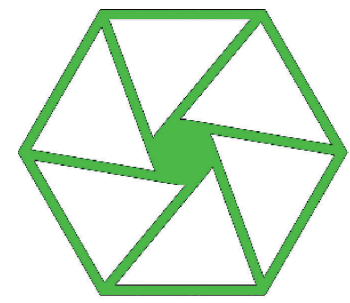

(e)

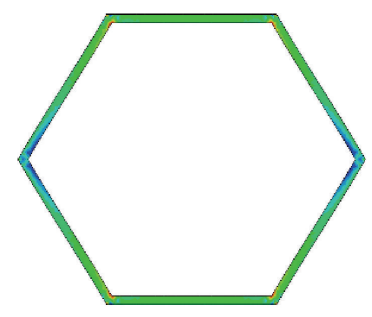

(b)

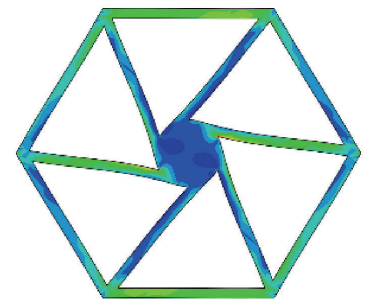

(f)

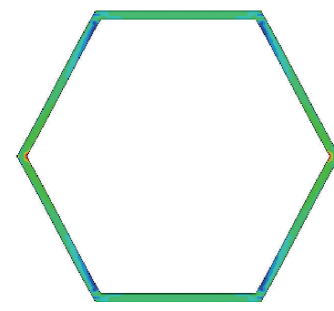

(c)

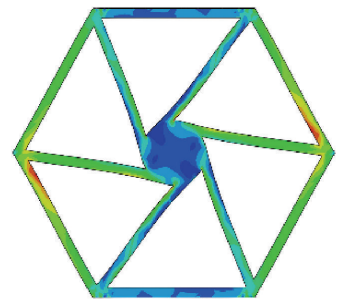

(g)

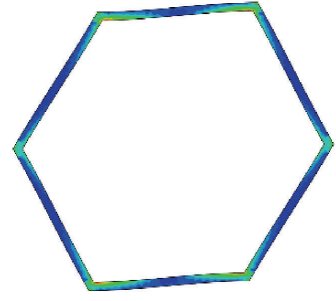

(d)

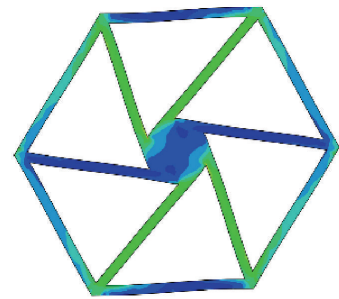

(h)

FIGURE 4: Schematic representation of loading conditions based on the listed cases in Table 1 (presented deformations are scaled larger than the actual ones for readability): (a) undeformed hexagonal RVE, (b) deformation under $e_{X X}^{\mathrm{M}}$, (c) deformation under $e_{Y Y}^{\mathrm{M}}$, (d) deformation under $e_{X Y}^{\mathrm{M}}$, (e) undeformed hexagonal-chiral RVE, (f) deformation under $e_{X X}^{\mathrm{M}}$, (g) deformation under $e_{Y Y}^{\mathrm{M}}$, and (h) deformation under $e_{X Y}^{\mathrm{M}}$.

TABLE 1: Loading conditions.

\begin{tabular}{lccc}
\hline Case & \multicolumn{3}{c}{ Imposed strains } \\
& $e_{X X}^{\mathrm{M}}$ & $e_{Y Y}^{\mathrm{M}}$ & $2 e_{X Y}^{\mathrm{M}}$ \\
\hline 1 & 0.01 & 0 & 0 \\
2 & 0 & 0.01 & 0 \\
3 & 0 & 0 & 0.01 \\
\hline
\end{tabular}

to be able to compare the numerical results with the closed form solutions provided for double cell walls in the literature $[5,9]$. The slenderness is taken to be in the range so that $\underline{t} / h \in[0.02,0.20]$ with increment of $\Delta t / h=0.02$ whereas hub diameter is taken as $D=h / 2$ for the chiral configuration illustrated in Figure 3(b).

3.2. Results. For understanding how cell wall slenderness (namely, cell wall thickness-to-height ratio) and cell topology (namely, chirality introduced inside the cell) affect the effective stiffness properties, simulation experiments are carried out on the RVEs. For this purpose, Abaqus/CAE is used, in which the periodic boundary conditions can be implemented. Thereafter, the solution domain is discretized into sets of finite elements-here, 6-noded quadratic triangular CPS6 provided in Abaqus/CAE. The results are compared with closed form solutions provided in Table 2.

3.2.1. Effect of Cell Wall Slenderness via Hexagonal RVE Simulations. Computational and analytical investigations on hexagonal RVEs are conducted so as to understand the effect of cell wall slenderness $t / h$ on the effective stiffness properties in the transverse plane. In case of regular hexagonal RVEs, cell walls are assumed to behave as mechanisms rather than rigid structures where the bending moments are the dominant deformation mechanisms under both uniaxial and shear loadings [18].

The comparison graphs presented in Figure 5 indicate that there is a positive effect of $t / h$ on the effective Young's moduli $E_{X}$ and $E_{Y}$ and shear modulus $G_{X Y}$. However, with increase in $t / h$, there is a decrease in Poisson's ratio values $v_{X Y}$ and $v_{Y X}$. In addition to the comparative studies, it is also deduced that Surveys I and II for regular hexagonal unit cells are in accordance with the computational homogenization results. This validates the current model and can hence be implemented on the proposed hexagonal-chiral configuration, which is elaborated in the following section.

3.2.2. Effect of Topology. For understanding the topological effects on the effective in-plane elastic parameters, closed form equations for regular hexagonal, square, and equilateral triangular unit cells listed in Table 2 are compared with the current computational investigations on hexagonal-chiral RVEs. The results plotted for effective moduli $E_{X}, E_{Y}$, and $G_{X Y}$ in Figure 6 show that stiffness is favored as the topology changes from hexagonal to triangular RVE. As stated in $[3,11]$, the main reason is the increasing dominancy of stretching over bending with this type of topological change. As also seen in Figure 6, the proposed hexagonal-chiral configuration results in much higher effective Young's moduli compared to regular hexagonal RVE. For instance, the ratio between the effective Young's moduli of two configurations for $\underline{t} / h=$ 0.20 is $\sim 100$, favoring the use of chirality. According to Figure 6, the hexagonal-chiral configuration yields the highest shear moduli among all of the investigated topologies. It is a promising result especially for the materials subject to shear loads-for example, packaging products and cargo containers. 
TABLE 2: Effective Young's moduli, shear modulus, and Poisson's ratios as functions of cell wall slenderness $\underline{t} / h$, where $\underline{t}=2 t$ for regular hexagonal, square, and equilateral triangular unit cells.

\begin{tabular}{|c|c|c|c|c|}
\hline Prop. & Survey I_HEX [5] & Survey II_HEX [9] & Survey III_SQU [5] & Survey IV_TRI [5] \\
\hline$E_{X}$ & $4 \quad(\underline{t} / h)^{3}$ & $(\underline{t} / h)^{3}$ & $\underline{t}$ & $2 \sqrt{3} \underline{t} / h+(\underline{t} / h)^{3}$ \\
\hline$\overline{E_{s}}$ & $\overline{\sqrt{3}} \overline{1+3(\underline{t} / h)^{2}}$ & $\overline{\sqrt{3}} \overline{1+\left(5.4+1.5 v_{s}\right)(\underline{t} / h)^{2}}$ & $\overline{\bar{h}}$ & $2 \sqrt{3+(t / h)^{2}}$ \\
\hline$E_{Y}$ & $4 \quad(\underline{t} / h)^{3}$ & $4 \quad(\underline{t} / h)^{3}$ & $\underline{t}$ & $2 \sqrt{3} \underline{t} / h+(\underline{t} / h)^{3}$ \\
\hline$\overline{E_{s}}$ & $\sqrt{\sqrt{3}} \overline{1+3(\underline{t} / h)^{2}}$ & $\overline{\sqrt{3}} \overline{1+\left(5.4+1.5 v_{s}\right)(\underline{t} / h)^{2}}$ & $\overline{\bar{h}}$ & $2 \sqrt{3+(t / h)^{2}}$ \\
\hline$G_{X Y}$ & $1 \quad(\underline{t} / h)^{3}$ & $(\underline{t} / h)^{3}$ & $1(\underline{t})^{3}$ & $\underline{\sqrt{3}} \underline{\underline{t}}+(\underline{\underline{t}})^{3}$ \\
\hline$E_{s}$ & $\sqrt{3} \overline{1+(\underline{t} / h)^{2}}$ & $\overline{\sqrt{3}} \overline{1+\left(3.3+1.75 v_{s}\right)(\underline{t} / h)^{2}}$ & $\frac{-}{2}(\bar{h})$ & $4 h(h)$ \\
\hline \multirow[b]{2}{*}{$v_{X Y}$} & $1-(\underline{t} / h)^{2}$ & $1+\left(1.4+1.5 v_{s}\right)(\underline{t} / h)^{2}$ & \multirow[b]{2}{*}{0} & $1-(\underline{t} / h)^{2}$ \\
\hline & $\overline{1+3(\underline{t} / h)^{2}}$ & $1+\left(5.4+1.5 v_{s}\right)(\underline{t} / h)^{2}$ & & $\overline{3+(t / h)^{2}}$ \\
\hline \multirow{2}{*}{$v_{Y X}$} & $1-(\underline{t} / h)^{2}$ & $1+\left(1.4+1.5 v_{s}\right)(\underline{t} / h)^{2}$ & \multirow{2}{*}{0} & $\underline{1-(\underline{t} / h)^{2}}$ \\
\hline & $\overline{1+3(t / h)^{2}}$ & $\overline{1+\left(5.4+1.5 v_{s}\right)(\underline{t} / h)^{2}}$ & & $\overline{3+(t / h)^{2}}$ \\
\hline
\end{tabular}
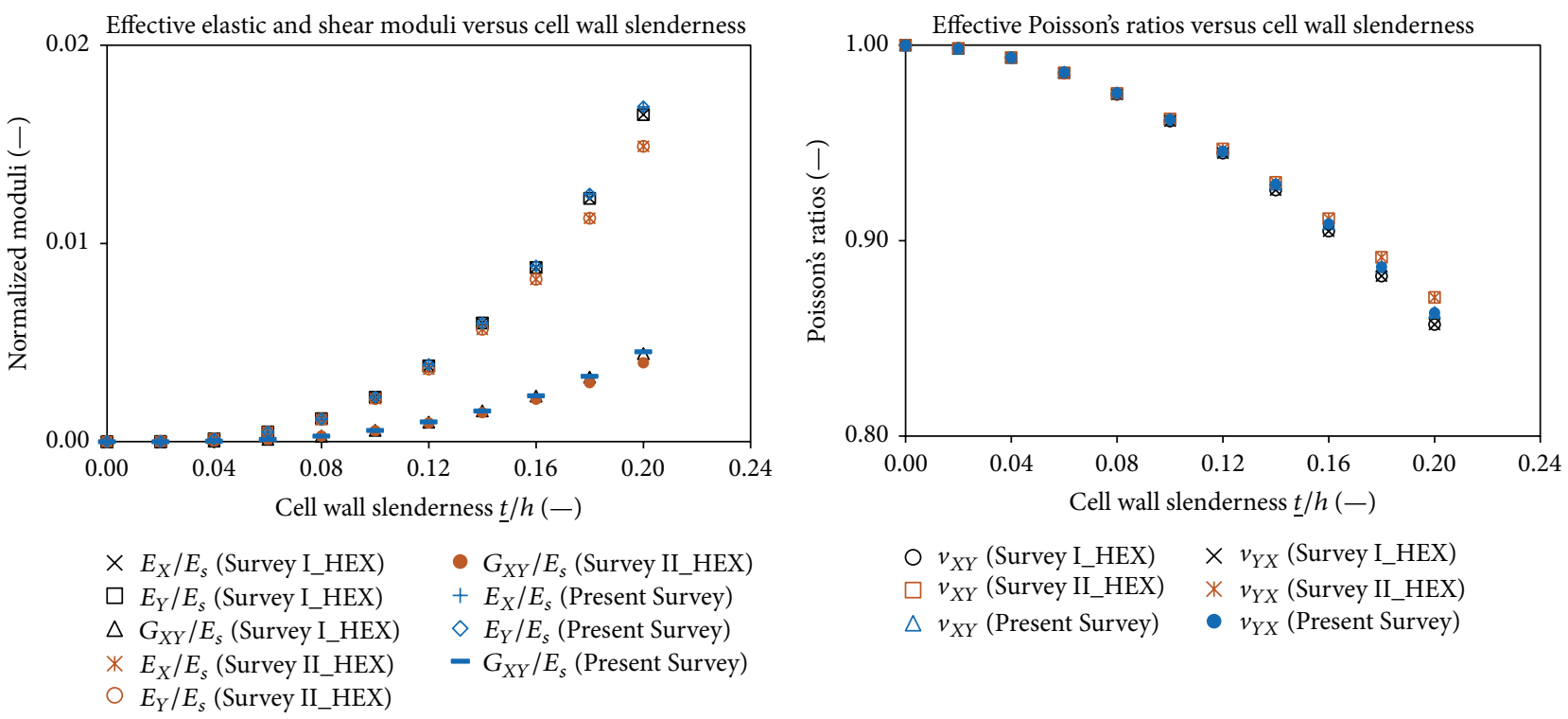

$\begin{array}{ll}\circ v_{X Y} \text { (Survey I_HEX) } & \times v_{Y X} \text { (Survey I_HEX) } \\ \square v_{X Y} \text { (Survey II_HEX) } & * v_{Y X} \text { (Survey II_HEX) } \\ \triangle v_{X Y} \text { (Present Survey) } & \bullet v_{Y X} \text { (Present Survey) }\end{array}$

FIGURE 5: Comparison of effective elastic parameters based on analytical and computational homogenization results.

Comparison of effective Poisson's ratios $v_{X Y}$ and $v_{Y X}$ in Figure 7 indicates that the regular hexagonal RVE has the highest linear elastic response-in terms of stretching or contraction-to the loading in the perpendicular direction. However, this response is lower for the triangular RVE and almost negligible for square one. The proposed hexagonalchiral configuration has $v_{X Y}$ and $v_{Y X}$ values that are much lower than the regular hexagonal RVE, which are comparable with those for the triangular and square RVEs.

\section{Conclusions}

In the present study, topological and morphological effects on the effective stiffness of cellular materials in the transverse plane are investigated by means of a first-order strain-driven computational homogenization method. For this purpose, representative volume elements (RVEs) of regular hexagonal, square, triangular, and hexagonal-chiral configurations are studied to understand how cell topology (i.e., nominal versus chiral) and cell wall slenderness affect the effective stiffness properties. Closed form solutions for regular hexagonal, square, and triangular RVEs provided in the literature are then taken as the basis for model validation and comparison. The results show that there is a positive effect of cell wall slenderness $t / h$ on the effective elastic parameters due to increase in the cell wall material volume. In addition to this, it is also deduced that there are drastic differences between regular hexagonal and hexagonal-chiral configurations, which can be explained in terms of deformation mechanism transformations between bending and stretching due to topological changes. Hence, the introduced chirality can be used as a tailored solution for materials and structures subject to high shear loads. 

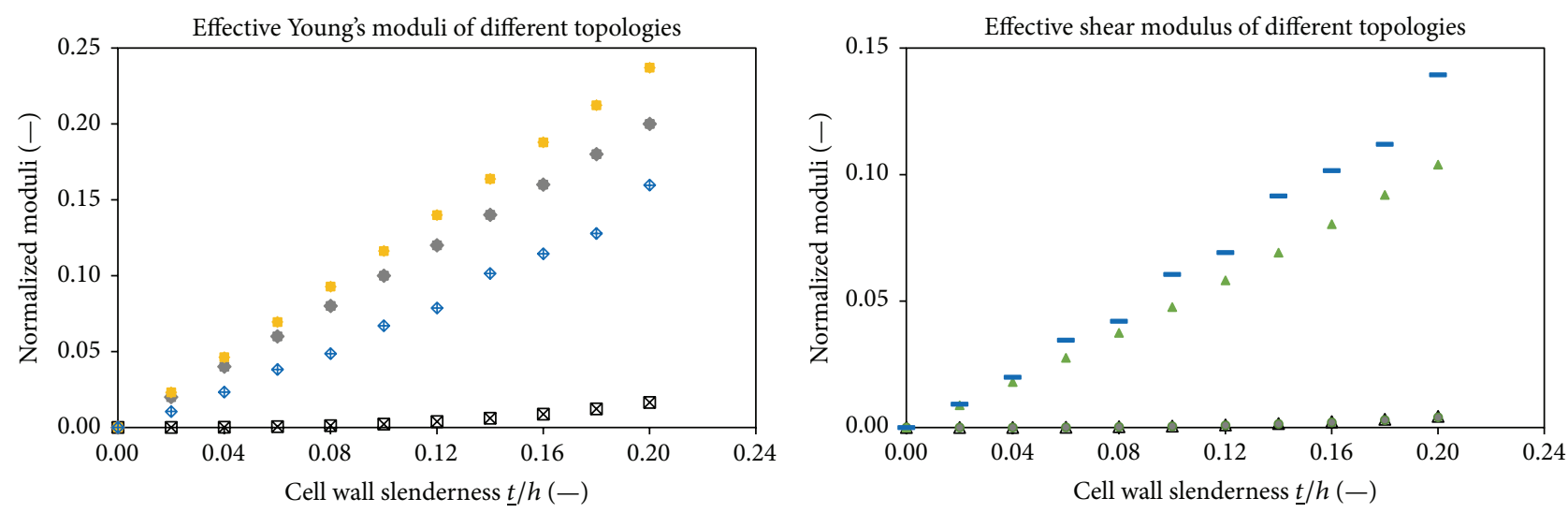

$$
\begin{aligned}
& \times E_{X} / E_{s} \text { (Survey I_HEX) } \quad E_{X} / E_{s} \text { (Survey IV_TRI) } \\
& \square E_{Y} / E_{s} \text { (Survey I_HEX) } \square E_{Y} / E_{s} \text { (Survey IV_TRI) } \\
& \bullet E_{X} / E_{s} \text { (Survey III_SQU) }+E_{X} / E_{s} \text { (Present Survey) } \\
& \text { - } E_{Y} / E_{s} \text { (Survey III_SQU) } \diamond E_{Y} / E_{s} \text { (Present Survey) }
\end{aligned}
$$

$$
\begin{aligned}
& \Delta G_{X Y} / E_{s} \text { (Survey I_HEX) } \quad \Delta G_{X Y} / E_{s} \text { (Survey IV_TRI) } \\
& -G_{X Y} / E_{s} \text { (Survey III_SQU) } \quad-G_{X Y} / E_{s} \text { (Present Survey) }
\end{aligned}
$$

Figure 6: Comparison of effective elastic and shear moduli based on analytical and computational homogenization results.

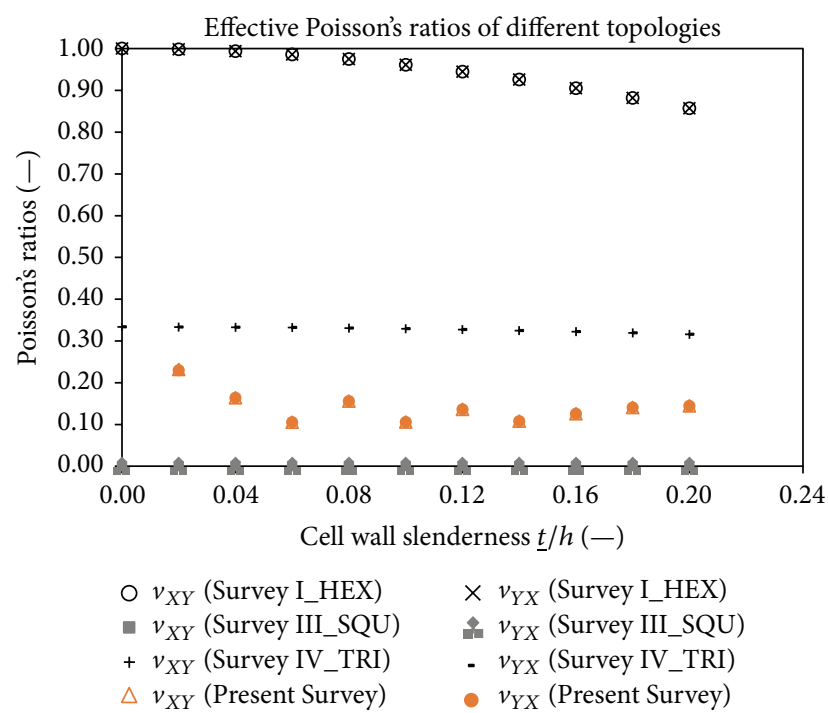

FIGURE 7: Comparison of effective Poisson's ratios based on analytical and computational homogenization results.

\section{Disclosure}

Any opinions, findings, and conclusions or recommendations expressed in this material are those of the authors and do not necessarily reflect the views of the sponsors.

\section{Competing Interests}

The authors declare that they have no competing interests.

\section{Acknowledgments}

The first author acknowledges the financial support of Tekniikan edistämissäätiö (TES) through Foundations' Post Doc Pool. The second author acknowledges support by the
National Science Foundation under Grant no. 1031181 (Program Managers Dr. Glaucio Paulino and Dr. Martin Dunn) and by the California Energy Commission's Energy Innovations Small Grants Program under Grant no. 09-03TE (Contract no. 500-98-014, Program Managers Stacie Ishida and David Chambers).

\section{References}

[1] A. Rafsanjani, Multiscale Poroelastic Model: Bridging the Gap from Cellular to Macroscopic Scale, ETH Zurich, Zurich, Switzerland, 2013.

[2] R. Harb, E. Taciroglu, and N. Ghoniem, "Partitioning of elastic energy in open-cell foams under finite deformations," Acta Materialia, vol. 61, no. 5, pp. 1454-1468, 2013.

[3] A. Karakoç, K. Santaoja, and J. Freund, "Simulation experiments on the effective in-plane compliance of the honeycomb materials," Composite Structures, vol. 96, pp. 312-320, 2013.

[4] M. G. D. Geers, V. G. Kouznetsova, and W. A. M. Brekelmans, "Multi-scale computational homogenization: trends and challenges," Journal of Computational and Applied Mathematics, vol. 234, no. 7, pp. 2175-2182, 2010.

[5] J. Freund, A. Karakoç, and J. Sjölund, "Computational homogenization of regular cellular material according to classical elasticity," Mechanics of Materials, vol. 78, pp. 56-65, 2014.

[6] V.-D. Nguyen, E. Béchet, C. Geuzaine, and L. Noels, "Imposing periodic boundary condition on arbitrary meshes by polynomial interpolation," Computational Materials Science, vol. 55, pp. 390-406, 2012.

[7] S. Nemat-Nasser and M. Hori, Micromechanics: Overall Properties of Heterogeneous Materials, North-Holland Publishing, Amsterdam, The Netherlands, 1999.

[8] J. Sjölund, A. Karakoç, and J. Freund, "Effect of cell geometry and material properties on wood rigidity," International Journal of Solids and Structures, vol. 62, pp. 207-216, 2015.

[9] L. Gibson and M. Ashby, Cellular Solids: Structure and Properties, Cambridge University Press, Cambridge, UK, 1999.

[10] A.-J. Wang, R. S. Kumar, and D. L. McDowell, "Mechanical behavior of extruded prismatic cellular metals," Mechanics of 
Advanced Materials and Structures, vol. 12, no. 3, pp. 185-200, 2005.

[11] M. Alkhader and M. Vural, "Mechanical response of cellular solids: role of cellular topology and microstructural irregularity," International Journal of Engineering Science, vol. 46, no. 10, pp. 1035-1051, 2008.

[12] J. Sjölund, A. Karakoç, and J. Freund, "Accuracy of regular wood cell structure model," Mechanics of Materials, vol. 76, pp. 35-44, 2014.

[13] E. Kahle and J. Woodhouse, "The influence of cell geometry on the elasticity of softwood," Journal of Materials Science, vol. 29, no. 5, pp. 1250-1259, 1994.

[14] F. Fritzen and T. Bohlke, "Influence of the type of boundary conditions on the numerical properties of unit cell problems," Technische Mechanik, vol. 30, pp. 354-363, 2010.

[15] K. Pham, V. G. Kouznetsova, and M. G. D. Geers, “Transient computational homogenization for heterogeneous materials under dynamic excitation," Journal of the Mechanics and Physics of Solids, vol. 61, no. 11, pp. 2125-2146, 2013.

[16] A. Karakoç and J. Freund, "A direct simulation method for the effective in-plane stiffness of cellular materials," International Journal of Applied Mechanics, vol. 5, no. 3, Article ID 1350034, 2013.

[17] A. Laghdir, Y. Fortin, C. M. De La Cruz, and R. E. Hernández, "Development of a technique to determine the 3D elasticity tensor of wood as applied to drying stress modeling," Maderas: Ciencia y Tecnologia, vol. 10, no. 1, pp. 35-44, 2008.

[18] V. S. Deshpande, M. F. Ashby, and N. A. Fleck, "Foam topology: bending versus stretching dominated architectures," Acta Materialia, vol. 49, no. 6, pp. 1035-1040, 2001. 

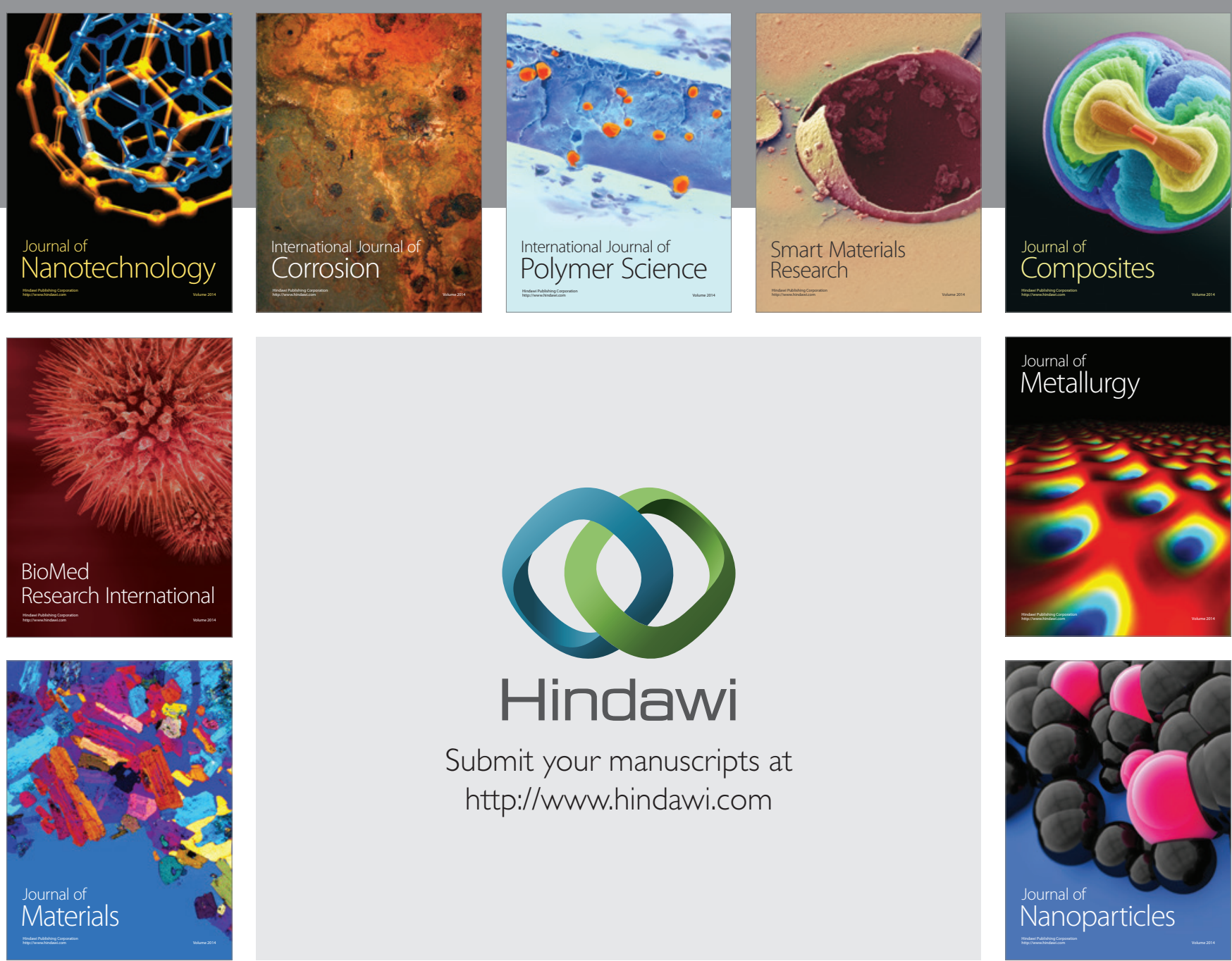

\section{Hindawi}

Submit your manuscripts at

http://www.hindawi.com

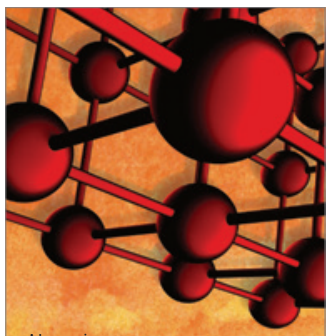

Materials Science and Engineering
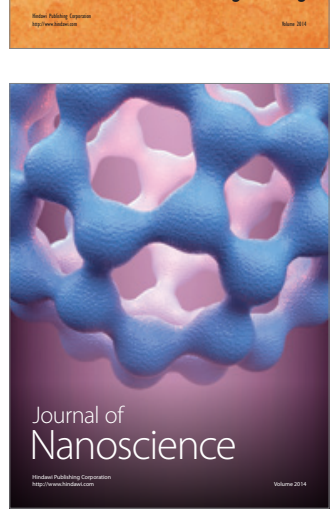
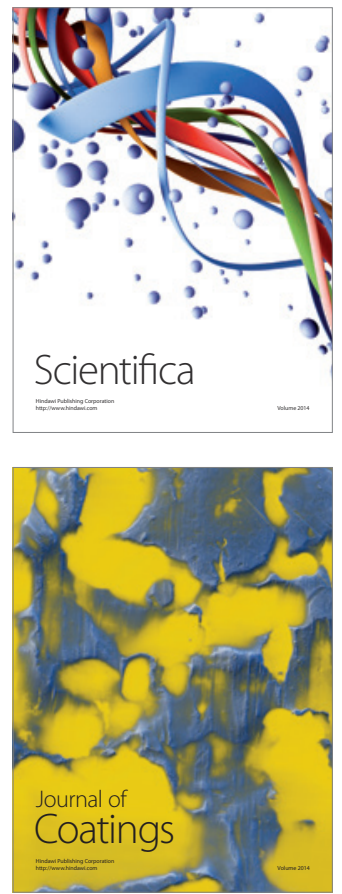
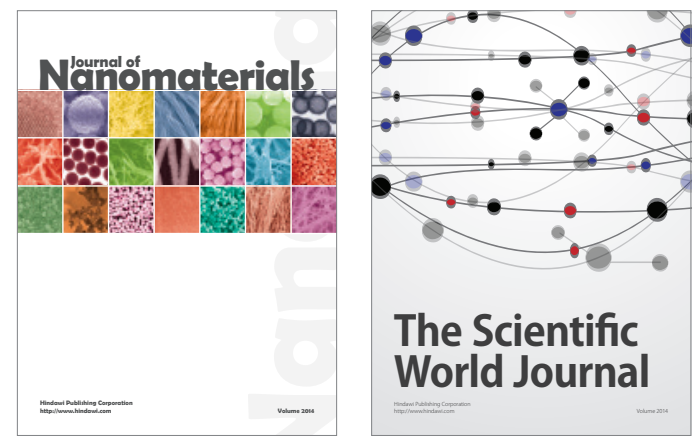

The Scientific World Journal
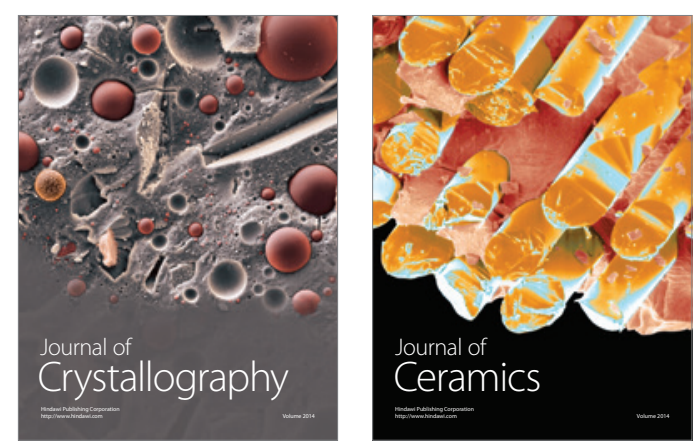
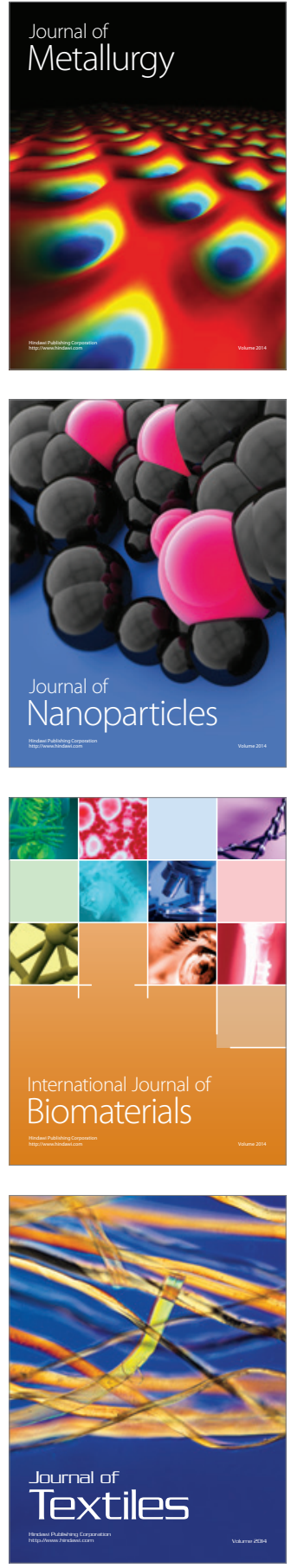